during the flooding associated with Hurricane Katrina, and this could be explained by the correlation we observe between the location of breach points and the high rate of subsidence beneath these levee sections (see supplementary information). Our subsidence estimates are probably minimum values considered over the lifetime of the levees, given that subsidence was most rapid in the first few years after their construction in the 1960s. Levee failure may have resulted from overtopping because the levees were too low - data collected after the storm ${ }^{5}$ indicate that water levels exceeded those expected by $0.9-1.7 \mathrm{~m}$. Alternatively, the high subsidence rates we observe might reflect active faulting or a weak, easily compacted substrate, promoting failure at or near the levee base. Timothy H. Dixon ${ }^{\star}$, Falk Amelung ${ }^{\star}$, Alessandro Ferretti†, Fabrizio Novali†, Fabio Roccat, Roy Dokkas, Giovanni Sella||, Sang-Wan Kim*, Shimon Wdowinski๋, Dean Whitman ๆ

${ }^{\star}$ RosenstielSchool of Marine and Atmospheric Science, University of Miami, Miami, Florida 33149, USA

e-mail:tdixan@rsmas.miami.edu

†Tele-Rilevamento Europa, 20149 Milan, Italy †Dipartimento di Elettronica e Informazione, Politecnico di Milano, 20133 Milan, Italy \$Center for Geolnformatics, Louisiana State University, Baton Rouge, Louisiana 70803, USA ||National Geodetic Survey, Silver Spring Maryland 20910-3282, USA
ๆDepartment of EarthSciences, Flarida

International University, Miami, Florida 33199, USA

1. Miller, L \& Douglas, B.C. Nbture 248,407-409 (2004)

2. Dolka, R.K.Gealogy 34, 281-284 (2006).

3 Shinkle, K. D. \&Dokka, R. K NOAA Tech. Pep. 50, 135 (2004)

4. Burkett, V. R, Zilkoski, D. B.\& Hart, D. in US Geal. Sun Subsidence int. GrpConf, Proc. Tech Meet, Galveston, Texas Nox 27-29, 2001 (eds Prince, K.R.\& Galloway, D. L) 63-70 (report 0B-308, US Geol Surx Water Resour Div, 200B).

5. Performance Evaluation of the New Orleans and Sautheast Lousiana Hurricane Protection System Rep. 2 (Interagency Per formance Evaluation Task Force, 2006).

Supplementary information accompanies this communication on Nature's website

Recelved 28 January; accepted 12 April 2006. Competing financial interests: declared (see online communication). doi:10.1038/441587a

\title{
SUPERFLUID HELIUM
}

\section{Visualization of quantized vortices}

When liquid helium is cooled to below its phase transition at $2.172 \mathrm{~K}$, vortices appear with cores that are only ångströms in diameter, about which the fluid circulates with quantized angular momentum ${ }^{1}$. Here we generate small particles of solid hydrogen that can be used to image the cores of quantized vortices in their three-dimensional environment of liquid helium. This technique enables the geometry and interactions of these vortices to be observed directly.

Since the discovery of quantized vortices ${ }^{2}$, attempts have been made to visualize them. Although the ends of parallel vortices in an array could be located $^{3}$, there has been no successful imaging of vortices in arbitrary three-dimensional configurations. Suspended par-
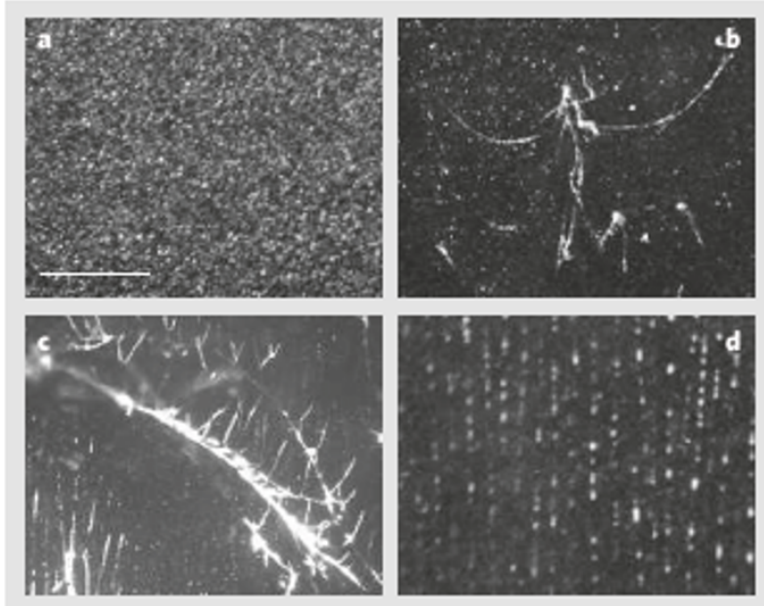

Figure 1 | Quantized vor tex cores in liquid helium. a-d, Images of particles (light against dark background) obtained with a camera and 105 -mm lens under different conditions: $a$, just above the transition temperature, when they are uniformly dispersed; b,c, on branching filaments at tens of millikelvin below the transition temperature, and d, regrouping along vertical lines for steady rotation about the vertical axis. In b and c, the particles on lines are evenly separated in small regions. Scale bar, $1 \mathrm{~mm}$.

tides can trace fluid motions, and the velocities of frozen particles in superfluid helium have been measured since hydrogen was first condensed for the purpose. These particles usually have diameters larger than $10 \mu \mathrm{m}^{5,6}$, although smaller polymer particles have been used? ${ }^{\text {? }}$.

Our technique generates smaller hydrogen particles by injecting a premixed gaseous solution of hydrogen, greatly diluted with helium, into liquid helium in its normal phase above the transition temperature (for details of methods, see supplementary information). This procedure yields a mist of randomly distributed hydrogen particles (Fig. 1a) that are smaller than the $2.7-\mu \mathrm{m}$ resolution of our long-range microscope. The suspension so prepared is then cooled to below the transition temperature.

Images taken with a digital camera focused on a thin laser-illuminated sheet show that not only do the particles trace fluid motions, but a fraction of them also collect on slender filaments, which are often several millimetres long (Fig. 1b, c).

Particles respond in a complicated way to superfluid flows ${ }^{s}$ and can be trapped in vortex cores? . The following evidence suggests that the observed filaments are particles collected on such cores. First, these filaments appear only below the transition temperature. Second, when the liquid-helium cell is set in steady rotation, the particles arrange themselves along uniformly spaced lines (Fig. 1d). The lines are parallel to the axis of rotation, which is in the image plane. This observation agrees with the expectation that quantized vortices form a rectilinear array aligned with the axis ${ }^{1}$. Third, if we assume that our sheet illuminates a slice of such an array, we find that the number density of lines per unit area normal to the axis of rotation, for a series of rotation rates, is consistent with Feynman's rule ${ }^{10}$, which predicts about $2,000 \Omega$ lines per $\mathrm{cm}^{2}$, where $\Omega$ is the angular velocity of the container in radians per second.

The filaments are complex: for example, they may give rise to branched networks and to particles evenly spaced along lines. Others have speculated how particles could act as passive tracers of the flow ${ }^{7.8}$; our images indicate that the presence of particles in the superfluid may transform the topology of vortex tangles by stabilizing forks in the vortices. This technique offers a tantalizing glimpse of new phenomena for future investigation.

Gregory P. Bewley*t, Daniel P. Lathrop*,

Katepalli R. Sreenivasan ${ }^{\star}+$

*Department of Physics, Institute for Research in Electronics and Applied Physics, Institute for Physical Sciences and Technology, University of Maryland, College Park, Maryland 20742, USA e-mait: dpl@complex.umd.edu

tDepartment of Mechanical Engineering, Yale University, New Haven, Connecticut 06520, USA †े Intemational Centrefor Theoretical Physics, Trieste 34014, Italy

1. Donnelly, R L Quantized Wortices in Hellum II (Cambridge Univ. Press, Cambridge, 1991).

2. Onsager, L Proc. Int. Conf Theox. Phys. 877-880 (Science Councilio of lapan, Tokyo, 1953).

3 Yarmchuk, E.J. Gordon, M.L V. \& Packard, R. E Phys. Rex Lett. 43, 214-218 (1979).

4. Chopra, K. L.\& Brown, L. B. Phys Rev. 108, 157 (1957).

5. Chung D. Y.\& Critchlow, P. R. Phys Rev.Lett. 14, 892-894 (1965).

6. Murakami M. \&lchilkawa, N. Onogenics 29, 438-443 (1989)

7. Zhang T.\& Van Sciver S. W. Nature Phys 1, 36-38 (2005)

8. Poole, D.R, Barenghi, C F, Sergeev, Y. A.\& Vinen, W. F. Phys. Rex B 71, 064514-1-16 (2005).

9. Parks, P.E.\&Donnelly, R. L.Phys Rev. Lett. 16, 45-48(1966).

10. Feynman R.P. in Prog Low Temp. Phys I (ed.Gorter, C. L) 17-53(North Holland Amsterdam, 1955)

Supplementary information accompanies this communication on Nature's website.

Recelved 23December 2005; accepted28 A pril 2006. Competing financial interests: declared none. doi:10.1038/441588a

BRIEF COMMUNICATIONS ARISING online www.nature.com/bca see Nature contents. 